\title{
System identification of spiking neuron networks: a model-driven approach
}

\author{
Daniele Linaro ${ }^{1}$, Marco Storace ${ }^{1}$, Maurizio Mattia ${ }^{2^{*}}$ \\ From Twentieth Annual Computational Neuroscience Meeting: CNS*2011 \\ Stockholm, Sweden. 23-28 July 2011
}

Understanding computational capabilities of the nervous system means to "identify" its emergent multiscale dynamics, possibly starting from the properties of its building blocks and following a "bottom-up" approach.

Here, we pursue this objective by adopting a model-driven identification, which we test on sparsely connected populations of excitatory integrate-and-fire (IF) neurons. Model neurons incorporate a fatigue mechanism underlying spike frequency adaptation (SFA) to lower discharge rates following a transient depolarization of the cell membrane potential [1]. We inspect the spontaneous relaxation time course of the population discharge rate without resorting to any linearization. The nonlinearity of the activity dynamics is exploited by aspecific suprathreshold stimulations in order to have a complete description of the system. This approach differs from those routinely employed in the study of the linear response properties of biological systems [2]. Furthermore, the network is investigated as a whole, the opposite of a bottom-up approach characterizing the system starting from its microscopic elements. Such description depicts the network dynamics in the low dimensional state space of the instantaneous discharge rate and the fatigue level of the adaptation mechanism, as suggested by a recent mean-field theory development [3].

Namely, from the elicited transient responses we work out the vector field of the reduced dynamics, and, based on the adopted theoretical framework, we extract the inputoutput gain function of the neurons in the network. The method allows testing the existence of an activity-dependent relaxation timescale for the network dynamics as expected from recent theoretical developments, extending the standard rate model framework $a$ la Wilson and

\footnotetext{
* Correspondence: maurizio.mattia@iss.it

${ }^{2}$ Department of Technologies and Health, Istituto Superiore di Sanità, Rome, Italy

Full list of author information is available at the end of the article
}

Cowan [4]. Besides, our model-driven identification makes available direct links to the microscopic level: indeed, we show how the decay time constant of the SFA, together with the absolute refractory period and the average synaptic efficacy, can be faithfully extracted. The robustness and generality of the introduced methodology is tested on relatively simple and well controlled in silico experiments, reporting a good agreement between theoretically expected and identified dynamics, even when phase transitions occur.

In conclusion, the devised identification has to be intended as a "middle-out" approach: starting from a mesoscopic description of the network dynamics, it provides in a "top-down" manner details about the microscopic domain at the cellular level. At the same time, the rate dynamics of a neuronal pool is quantitatively estimated, allowing for a "bottom-up" effective description of multi-modular macroscopic networks of neurons. The assumptions behind the underlying theoretical framework make the method of wide applicability to controlled biological preparations like cultured neuron networks and in vitro brain slices.

\section{Author details}

'Department of Biophysical and Electronic Engineering, University of Genoa, Genoa, Italy. ${ }^{2}$ Department of Technologies and Health, Istituto Superiore di Sanità, Rome, Italy.

Published: 18 July 2011

\section{References}

1. Koch C: Biophysics of Computation: Information Processing in Single Neurons. New York: Oxford University Press; 1999, 562

2. Sakai HM: White-noise analysis in neurophysiology. Physiological Reviews 1992, 72:491-505.

3. Gigante G, Mattia M, Del Giudice P: Diverse population-bursting modes of adapting spiking neurons. Physical Review Letters 2007, 98:148101.

4. Wilson HR, Cowan JD: Excitatory and inhibitory interactions in localized populations of model neurons. Biophysical Journal 1972, 12:1-24.

doi:10.1186/1471-2202-12-S1-P215

Cite this article as: Linaro et al:: System identification of spiking neuron networks: a model-driven approach. BMC Neuroscience 2011 12(Suppl 1): P215.
C Biomed Central

(c) 2011 Linaro et al; licensee BioMed Central Ltd. This is an open access article distributed under the terms of the Creative Commons Attribution License (http://creativecommons.org/licenses/by/2.0), which permits unrestricted use, distribution, and reproduction in any medium, provided the original work is properly cited. 